\title{
The role of autophagy in Paneth cell differentiation and secretion
}

\section{TS Stappenbeck ${ }^{1}$}

\begin{abstract}
Paneth cells are a small intestinal epithelial cell lineage that is considered to have a role in innate immune function. Recent studies on mice with diminished and/or loss of autophagy have suggested that Paneth cells are a primary target in vivo. Interestingly, loss of autophagy affects the secretion of antimicrobial proteins from Paneth cells. Understanding the intersection of the autophagy pathway with the secretory apparatus, which is a key feature of differentiation of Paneth cells, is a key unanswered question.
\end{abstract}

Paneth cells are a morphologically and geographically distinctive epithelial cell type located throughout the length of the small intestine in both mice and humans. Another location for these cells is the proximal human colon that occurs only as a metaplastic response to an inflammatory insult. In the small intestine, Paneth cells are one of the four lineages produced continuously by epithelial stem cells. ${ }^{1}$ These multipotent stem cells comingle with Paneth cells in the base of frequent epithelial invaginations called crypts of Lieberkühn. Only a few dozen Paneth cells populate each crypt. However, because of the substantial number of small intestinal crypts in a mouse $(\sim 1.1$ million), the overall number of Paneth cells is $>20$ million.

The localization of Paneth cells within the epithelial monolayer of the small intestine is distinct compared with that of the other three differentiated lineages that are produced by stem cells: absorptive enterocytes, mucus-producing goblet cells, and hormone-secreting enteroendocrine cells. These three lineages emerge from crypts and migrate onto evaginations of the mucosal surface into the lumen of the intestine called villus. Each crypt supplies these cells to multiple surrounding villi (each villus is supplied by 6-8 surrounding crypts). The lifespan of villus epithelial cells is relatively short (on the order of 3-5 days) as compared with Paneth cells that migrate to the base of crypts where they live much longer $(>30$ days).

Recent advances have been made in the identification of transcription factors and signaling pathways that influence the Paneth cell lineage specification and differentiation (see Stappenbeck ${ }^{2}$ for review). In the adult mouse small intestine, Wnt and Notch signaling pathways are dominant. These pathways are additionally important in maintaining the crypt structure and proliferation of stem/progenitor cells. Sox-9 is one of the key transcription factors that acts downstream of Wnt signaling to specify Paneth cells, as knockout of this gene leads to mice with crypts and active stem cells, but no Paneth cells. Other transcription factors such as the ets factor, Spdef, which interact with both Wnt and Notch signaling pathways, are required for Paneth cell differentiation.

The location of Paneth cells in the crypt base is likely to be strategically important to their highly specialized function, which is to produce and secrete specified proteins. In the small intestine, Paneth cells are a critical source of cationic antimicrobial proteins that are delivered to the lumen of the gut by a process of merocrine secretion through their apical plasma membrane. This aspect of secretion is by far the most highly studied in these cells. ${ }^{3}$ Interest in this process has been driven by histological observations first made in the late 19th century that showed that these cells have very distinctive cytoplasmic granules. These granules can be highlighted on sections of small intestine prepared for light microscopy that are stained with either periodic acid Shiff or various lectins including Ulex europaeus agglutinin 1 . In addition, sections prepared for transmission electron microscopy show the well-described electron-dense secretory granules (Figure 1). Biochemical and immunohistochemical studies have shown that these secretory granules store various small molecular weight antimicrobial proteins, including a variety of $\alpha$-defensins (cryptdins), angiogenin-4, lysozyme, secretory phospholipase A2, and Reg3 $\gamma$. Similar to most

1'Department of Pathology and Immunology Washington University School of Medicine, St Louis, MO, USA. Correspondence: TS Stappenbeck (stappenb@pathology. wustl.edu) 


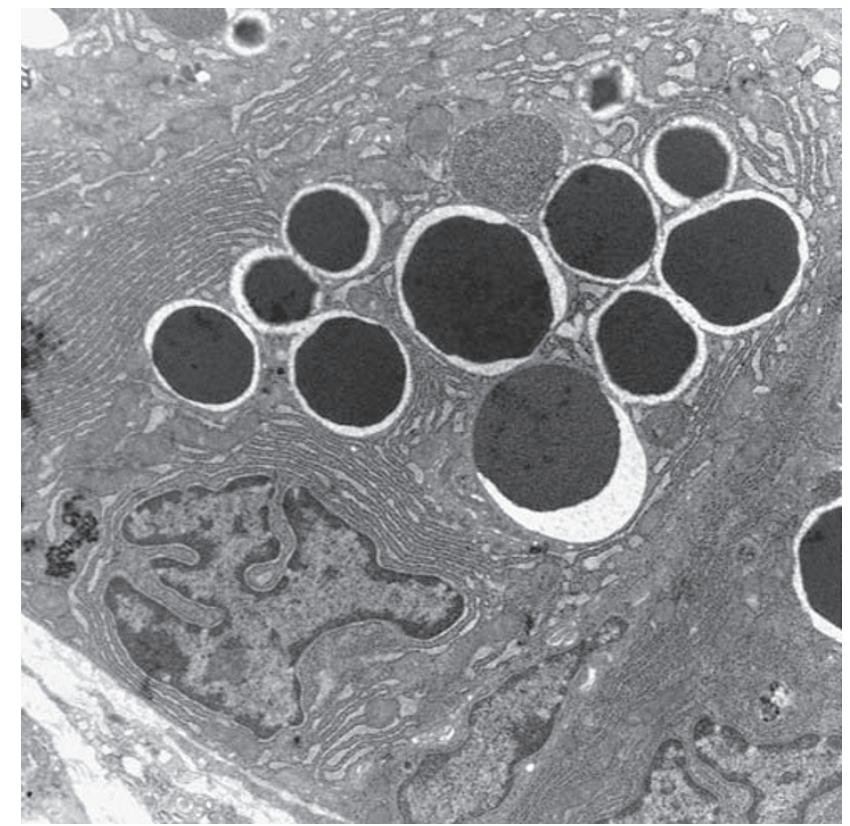

Figure 1 "Good" Paneth cells. Transmission electron micrograph of small intestinal Paneth cells from a wild-type mouse. Note the electron-dense secretory granules and layered endoplasmic reticulum.

highly secretory cells, Paneth cells additionally contain a robust rough endoplasmic reticulum and Golgi apparatus that act as part of the "factory" to produce these proteins.

The production of antimicrobial proteins by Paneth cells seems to have functional consequences in both health and disease. These antimicrobial proteins likely shape the composition and abundance of the species of indigenous microbes that reside within the intestinal lumen. Cryptdins produced by Paneth cells have also been shown to have a role in the clearance of pathogens. In one example, mice that were engineered to overexpress one member of the cryptdin family of proteins were much less susceptible to oral infection by Salmonella typhimurium. ${ }^{4}$

Paneth cells also secrete proteins that influence other functions in the intestine. The Wnt ligands that they produce likely affect stem cells in the crypt base. They also produce factors that influence villus vasculature development. Paneth cell also produce inflammatory cytokines such as tumor necrosis factor- $\alpha$ (reviewed by Stappenbeck ${ }^{2}$ ). It is not known whether these factors are secreted apically or basolaterally. The ability to now grow intestinal crypts in culture will aid in the study of the mechanism of secretion of this cell type (for example, see Sato ${ }^{5}$ ).

Not only do Paneth cells potentially alter the composition of the microbiome in the gut lumen but these microbes can influence Paneth cell gene expression and function as well (reviewed by Stappenbeck ${ }^{2}$ ). For example, one of the major bacterial species in the small intestine, Bacteroides thetaiotaomicron, stimulates the expression of an antimicrobial protein, angiogenin-4, in Paneth cells. This bacterium also stimulates angiogenesis in the mesenchymal core of small intestinal villi through Paneth cells. The Paneth cell-intestinal bacterial cross-talk is particularly interesting in light of recent findings that show that Paneth cells can limit bacterial penetration of intestinal microbes across the epithelial barrier. This function depends on Toll-like receptor-Myd88 signaling in Paneth cells.

The fact that Paneth cells regulate the ability of intestinal bacteria to pass through the epithelial barrier suggests that autophagy may be important in this cell type. Autophagy is a process that occurs in all cells and its major function is the recycling of intracellular components. ${ }^{6}$ The machinery that is required for this macro-autophagocytic process is complex and requires $>20$ different proteins to generate the classic double membrane-bound structures in the cytosol. These autophagocytic vesicles are then delivered to lysosomes for recycling of individual components. In some cell types, loss of autophagy function can lead to alterations in proliferation, cell death, and loss of the ability to clear intracellular pathogens. Paneth cells have been observed to form autophagocytic vesicles when cells are stressed by processes such as irradiation. ${ }^{7}$

Another reason to consider the role of autophagy in Paneth cells (as well as in other intestinal cell types) is that patients with one form of inflammatory bowel disease, Crohn's disease, have polymorphisms in specific genes, including the autophagy gene Atg16L1. This protein forms a complex with two other autophagy proteins, Atg5 and Atg12, which together function to extend the membrane of a nascent autophagocytic vesicle. Loss of autophagy function in fibroblasts through the manipulation of Atg16L1 function suggests that bacterial clearance is impaired because of loss of autophagy. ${ }^{8}$ However, loss-of-function studies in mice for Atg16L1 did not find a role in the clearance of enteric pathogenic bacteria, including Salmonella and Listeria. ${ }^{9}$

Instead, mice with loss of function for Atg16L1 showed defects in the secretory function of Paneth cells. Autophagydeficient Paneth cells properly committed to differentiation and did not seem to undergo premature apoptosis. However, diminished autophagy function in Paneth cells led to defects in the packaging of antimicrobial proteins into granules and eventually their proper export into the lumen of the gut is defective. ${ }^{9}$ Instead of a robust endoplasmic reticulum and secretory vesicles, the autophagy-deficient Paneth cell cytoplasm contained few granules and was typically filled with small vesicles (Figure 2). The nature of these cytoplasmic vesicles is still unclear. To test for the possibility that they might be endocytic vesicles that sample luminal bacteria, we stained for bacteria on tissue sections. However, we have thus far found no evidence that these vesicles contain bacteria. Microarray studies of these Paneth cells showed that in 


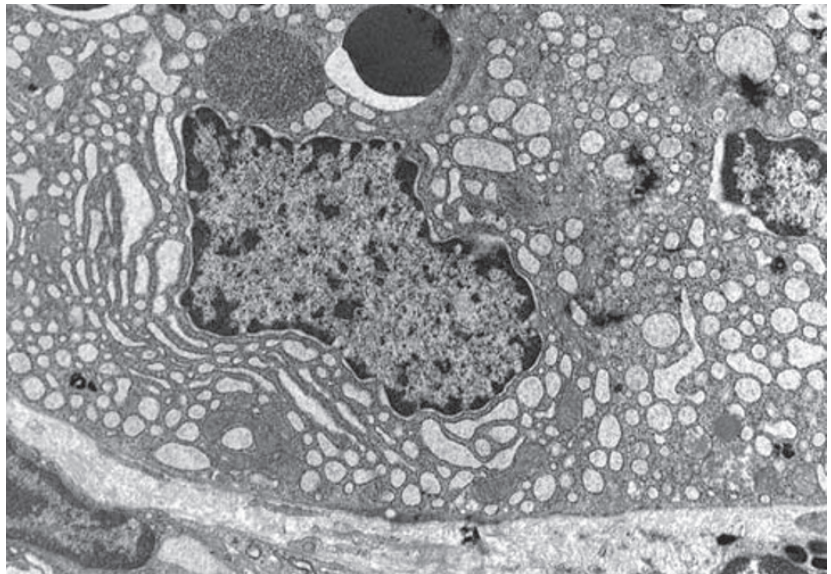

Figure 2 "Bad" Paneth cells. Transmission electron micrograph of small intestinal Paneth cells from an Atg16L1-deficient mouse. Note the loss of electron-dense secretory granules and layered endoplasmic reticulum that is replaced by cytoplasmic vesicles.

addition to a defect in apical secretion, autophagy-deficient Paneth cells showed an altered expression of inflammatory cytokines such as tumor necrosis factor$\alpha$, leptin, and adiponection. The major signaling pathway that was enhanced in autophagy-deficient Paneth cells was PPAR (peroxisome proliferator-activated receptor pathway). The role of this family of signaling pathways is thought to be anti-inflammatory and PPAR $\beta / \delta$ has a role in Paneth cell differentiation. ${ }^{10}$ Thus, autophagy-deficient Paneth cells, which have severe alterations in secretion, may attempt to compensate for this defect by an enhancement of the expression of components of these pathways. We also found similar alterations in Paneth cells from Crohn's disease patients who were homozygous for the known ATG16L1 susceptibility allele, further showing the relevance of this mouse model to human pathology. Thus, the function of autophagy in Paneth cells seems to have a critical role in targeting antimicrobial proteins to secretory granules and in limiting the expression of inflammatory cytokines.

Many questions arise from these initial studies. Why is the function of Paneth cell secretion targeted by loss of autophagy? What is the role of PPARs in autophagydeficient Paneth cells? It will be critical to examine the role of the human susceptibility allele in mouse models to further study these questions. Another puzzle is that if such a large population of Americans are homozygous for the ATG16L1 susceptibility allele ( $\sim 1 / 3$ of the population), do all these people have defective Paneth cells or is there an environmental or second genetic hit that acts as a trigger for defective Paneth cells? Finally, are there additional intestinal epithelial cells that, similar to Paneth cells, have defective secretion in response to loss of autophagy? Paneth cells are a classic example of a highly secretory cell that produces a limited repertoire of specified proteins for a particular function. Other examples of highly secretory cells in the gastrointestinal tract include salivary gland acinar cells, zymogenic and parietal cells in the stomach, colonic goblet cells, pancreatic acinar and islet cells, and plasma cells. Each of these cells has a robust endoplasmic reticulum, Golgi, and secretory granules similar to Paneth cells. Many further studies will be required to evaluate the role of autophagy in these cell types.

\section{DISCLOSURE}

The author declared no conflict of interest.

C 2010 Society for Mucosal Immunology

\section{REFERENCES}

1. Cheng, H. \& Leblond, C.P. Origin, differentiation and renewal of the four main epithelial cell types in the mouse small intestine. IV Paneth cells. Am. J. Anat. 141, 521-536 (1974).

2. Stappenbeck, T.S. Paneth cell development, differentiation and function: new molecular cues. Gastroenterology 137, 30-33 (2009).

3. Ouellette, A.J. Paneth cells and innate immunity in the crypt microenvironment. Gastroenterology 113, 1779-1784 (1997).

4. Salzman, N.H., Ghosh, D., Huttner, K.M., Paterson, Y. \& Bevins, C.L. Protection against enteric salmonellosis in transgenic mice expressing a human intestinal defensin. Nature 422, 522-526 (2003).

5. Sato, T. et al. Single Lgr5 stem cells build cryptvillus structures in vitro without a mesenchymal niche. Nature 459, 262-265 (2009).

6. Levine, B. \& Kroemer, G. Autophagy in the pathogenesis of disease. Cell 132, 27-42 (2008).

7. Gorbunov, N.V. \& Kiang, J.G. Up-regulation of autophagy in small intestine Paneth cells in response to total-body gamma-irradiation. J. Pathol. 219, 242-252 (2009).

8. Kuballa, P., Huett, A., Rioux, J.D., Daly, M.J. \& Xavier, R.J. Impaired autophagy of an intracellular pathogen induced by a Crohn's disease associated ATG16L1 variant. PLOS ONE 3, e3391 (2008).

9. Cadwell, K. et al. A key role for autophagy and the autophagy gene Atg16/1 in mouse and human intestinal Paneth cells. Nature 456, 259-263 (2008).

10. Varnat, F. et al. PPARbeta/delta regulates paneth cell differentiation via controlling the hedgehog signaling pathway. Gastroenterology 131, 538-553 (2006). 